\title{
Sexual Harassment Is an Occupational Hazard
}

\author{
Sabine Oertelt-Prigione, $\mathrm{MD}, \mathrm{PhD}^{1,2}$
}

$\mathbf{S}$ INCE THE BEGINNING of the \#metoo movement about 2 years ago, medicine and health care are facing their own moment of reckoning. This should not come as a surprise. Sexual harassment is a pervasive phenomenon that can affect all areas of work, ${ }^{1}$ yet the field of medicine displays some peculiar risks. As physicians we have to invade our patients' privacy verbally and physically on a daily basis, just to do our job. However, this is only a small part of the problem. Historically, the practice of medicine was associated with a work culture that praised relentless drive and expected unlimited competence and infallibility. The ones that believed to have achieved this heroic status frequently also felt entitled to immunity and impunity. This applied 25 years ago, when the Lancet published an editorial addressing the "professional disgrace" of harassment ${ }^{2}$ and is often still true today. This is the larger part of the problem.

In a context of perfectionism and endurance, the human dimension of work was often ignored, as if social relations were a by-product of work and not an essential grounding aspect of it. The problem was framed according to three misconceptions. First, harassment was judged a minority issue affecting a few overly sensitive women unfit for the job. Second, if harassment was indeed identified, the perpetrators had to be a few "rotten apples" within the organization. Third, harassment was not supposed to cause any physical or psychological harm and, as such, had no priority.

All three of these assumptions are false as the article by Vargas et al. published in this issue ${ }^{3}$ of the journal once again demonstrates. The authors report on a faculty-wide survey at University of Michigan Medical School in which participants recounted their experiences with sexual harassment in the workplace.

The first assumption is that only a small number of women are affected. Emerging data, as the one Vargas et al. ${ }^{3}$ are reporting in this issue, are contradicting the long-held belief that sexual harassment is a niche problem. Many former studies, including the recent landmark report by the National Academies, ${ }^{4}$ solely concentrated on women as victims. Although women are more frequently affected, limiting a study to their experiences does not allow grasping the magnitude of the problem. Vargas reports that $60 \%-80 \%$ of the workforce-female and male-has experienced some incident in the past 12 months. This is in line with our own data from Germany, ${ }^{5}$ which also showed significant rates of harassment among men. Hence, we are not addressing a niche problem. Harassment affects the majority of the workforce and everyone is at risk.

The second assumption is that perpetrators within organizations have to be few, if present at all. If $60 \%-80 \%$ of the workforce is affected, the perpetrators cannot be few. Second, if employees are explicitly asked about the identity of perpetrators, insiders are most frequently mentioned, in Vargas et al. ${ }^{3}$ and other studies ${ }^{6}$ with the only exception of emergency rooms. ${ }^{7}$ It is important to acknowledge patients and their families as perpetrators, ${ }^{8}$ but the main source of harassment are insiders, colleagues, and superiors. We are not identifying a few individuals; we are seeing the widespread consequences of a culture that has established impunity, if not encouragement, for certain behaviors. And since newcomers will mimic the existing culture to integrate into the system, these behaviors will be perpetuated if not explicitly addressed and rejected.

The third assumption is that sexual harassment does not bear relevant consequences for the victim, and that the numbers are too low to require intervention (see assumption number one). Although the consequences of trauma are well studied, ${ }^{9}$ we lack the same depth of information about the specific consequences of sexual harassment. ${ }^{10}$ Vargas et al. ${ }^{3}$ report an association of lower mental health, job satisfaction, and feeling of safety at work with sexual harassment in both female and male participants. A cross-sectional study will not allow establishing causality, but these associations and patterns are recurring. Harassment has physical, mental, and occupational consequences ${ }^{11}$ and its long-term effects warrant further investigation.

Given all this data sexual harassment appears as a highly prevalent phenomenon that impacts the entire workforce and has serious health and occupational consequences. Sexual harassment is a severe occupational hazard. The first pillar of the Hippocratic oath is "do no harm." Sexual harassment is occupational harm. Next to the moral and ethical obligation of doing no harm, there is also a legal one. Employers have a legally mandated duty of care in most industrialized countries. As such, time, personnel, and economic resources should be deployed for the prevention of such an impactful phenomenon. Theoretically these resources should be allocated based on risk and impact. Considering, thus, the amount of resources invested in the prevention of radiation poisoning in relation to the relative risk of ever experiencing it, imagine how much time, personnel, and money could be invested in

\footnotetext{
${ }^{1}$ Department of Primary and Transmural Care, Radboud Institute of Health Sciences (RIHS), Radboud University Medical Center, Nijmegen, The Netherlands.

${ }^{2}$ Department of Legal and Forensic Medicine, Charité-Universitätsmedizin, Berlin, Germany.
} 
the prevention of a highly prevalent and toxic phenomenon. It is time to move the discourse from an individualized to an institutional level, from single cases to collective action. If we fail to do so, 30 years from now the "professional disgrace" will still be an inevitable reality for our profession.

\section{References}

1. International Labor Organization (ILO). Code of conduct and guidelines to prevent and address on sexual harassment in the workplaces. Ceylon: Employers' Federation of Ceylon (EFC) and International Labor Organization, 2013.

2. The Lancet. A professional disgrace. Lancet 1993;342:627628 .

3. Vargas E, Brassel S, Cortina L, Settles I, Johnson T, Jagsi R. \#MedToo: A large-scale examination of the incidence and impact of sexual harassment of physicians and other faculty at an academic medical center. J Womens Health 2019;29:13-20.

4. NASEM National Academies of Sciences EaM. Sexual harassment of women climate, culture, and consequences in academic sciences, engineering, and medicine. Washington, DC: The National Academies Press, 2018.

5. Jenner S, Djermester P, Prugl J, Kurmeyer C, OerteltPrigione S. Prevalence of sexual harassment in academic medicine. JAMA Intern Med 2019;179:108-111.
6. Crebbin W, Campbell G, Hillis DA, Watters DA. Prevalence of bullying, discrimination and sexual harassment in surgery in Australasia. ANZ J Surg 2015;85:905-909.

7. Shafran-Tikva S, Zelker R, Stern Z, Chinitz D. Workplace violence in a tertiary care Israeli hospital-A systematic analysis of the types of violence, the perpetrators and hospital departments. Isr J Health Policy Res 2017;6:43.

8. Nelson R. Tackling violence against health-care workers. Lancet 2014;383:1373-1374.

9. McFarlane AC. The long-term costs of traumatic stress: Intertwined physical and psychological consequences. World Psychiatry 2010;9:3-10.

10. Abbasi J. Sexual harassment and assault associated with poorer midlife health in women. JAMA 2019;321:234-236.

11. Willness CR, Steel P, Lee K. A meta-analysis of the antecedents and consequences of workplace sexual harassment. Pers Psychol 2007;60:127-162.

Address correspondence to:

Sabine Oertelt-Prigione, $M D, P h D$

Department of Primary and Transmural Care

Radboud Institute of Health Sciences (RIHS)

Radboud University Medical Center

Geert Grooteplein 21

Nijmegen $6500 H B$

The Netherlands

E-mail: sabine.oertelt-prigione@radboudumc.nl 